\title{
ON BI-INVARIANT WORD METRICS
}

\author{
ŚWIATOSŁAW R. GAL AND JAREK KElDA
}

\begin{abstract}
We prove that bi-invariant word metrics are bounded on certain Chevalley groups. As an application we provide restrictions on Hamiltonian actions of such groups.
\end{abstract}

\section{INTRODUCTION}

1.A. The result. Let $\mathcal{O}_{\bigvee} \subset \mathfrak{K}$ be a ring of $\bigvee$-integers in a number field $\mathfrak{K}$, where $V$ is a set of valuations containing all Archimedean ones. Let $\mathrm{G}_{\pi}\left(\Phi, \mathcal{O}_{\vee}\right)$ be the Chevalley group associated with a faithful representation $\pi: \mathfrak{g} \rightarrow \mathfrak{g l}(\mathrm{V})$ of a simple complex Lie algebra of rank at least two (see Section 4.A for details).

Theorem 1.1. Let $\Gamma$ be a finite extension or a supergroup of finite index of the Chevalley group $\mathrm{G}_{\pi}\left(\Phi, \mathcal{O}_{\vee}\right)$. Then any bi-invariant metric on $\Gamma$ is bounded.

The examples to which Theorem 1.1 applies include the following groups.

(1) $\operatorname{SL}(n ; \mathbf{Z})$; it is a non-uniform lattice in $\operatorname{SL}(n ; \mathbf{R})$.

(2) $\operatorname{SL}(n ; \mathbf{Z}[\sqrt{2}])$; the image of its diagonal embedding into the product $\mathrm{SL}(n ; \mathbf{R}) \times \mathrm{SL}(n ; \mathbf{R})$ is a non-uniform lattice.

(3) $\mathrm{SO}(n ; \mathbf{Z}):=\left\{\mathrm{A} \in \mathrm{SL}(n, \mathbf{Z}) \mid \mathrm{AJA}^{\mathrm{T}}=\mathrm{J}\right\}$, where $\mathrm{J}$ is the matrix with ones on the anti-diagonal and zeros elsewhere. It is a nonuniform lattice in the split real form of $\mathrm{SO}(n, \mathbf{C})$.

(4) $\operatorname{Sp}(2 n ; \mathbf{Z})$; it is a non-uniform lattice in $\operatorname{Sp}(2 n ; \mathbf{R})$. Its nontrivial central extension by the infinite cyclic group has unbounded bi-invariant word metric (see Example 3.11).

The details of these and other examples are presented in Section 4.C on page 12 .

1.B. Remarks. The proof of Theorem 1.1 follows from the boundedness of the bi-invariant word metric. This is the usual word metric induced by a set of generators invariant under conjugation. Such generating sets are in general infinite. However, if a group $\Gamma$ is generated by conjugates of finitely many elements then the Lipschitz 
equivalence class of such metrics is well defined and it is maximal among all bi-invariant metrics. In particular, if such a bi-invariant word metric is bounded then so is any bi-invariant metric.

Thus the proof amounts to showing that the bi-invariant word metric is bounded on a group $\Gamma$ as in Theorem 1.1. It is a combination of two known facts. The first is that the group $\mathrm{G}\left(\mathrm{O}_{V}\right)$ has bounded generation. This means that there is a subset $X \subset G\left(\mathcal{O}_{V}\right)$ and a number $m \in \mathbf{N}$ such that every element $g \in \mathrm{G}\left(\mathrm{O}_{\mathrm{V}}\right)$ is a product of at most $m$ elements from $X$. The second fact is that the bi-invariant word norm is bounded on X. The details are presented in Section 4.A.

Although the bounded generation is inherited by finite index subgroups, bi-invariant word metrics do not behave well with this respect. For example, $\mathbf{Z}$ is an index two unbounded subgroup in the infinite dihedral group that is bounded (Example 2.7). In Section 3, we present (mostly well known) tools used to prove unboundedness of bi-invariant word metrics.

Question 1.2. Suppose that $\mathrm{G}$ is semisimple real Lie groups of higher rank and with finite centre. Is a lattice $\Gamma \subset \mathrm{G}$ bounded with respect to the bi-invariant word metric?

Notice that certain lattices in groups of rank 1 admit nontrivial homogeneous quasi-homomorphisms which implies that their bi-invariant word metrics are unbounded (see Lemma 3.6).

The commutator length (on a perfect group), and its generalisation due to Calegari and Zhuang [4] called the W-length, as well as the torsion length (on a group generated by torsion elements) [16] induce bi-invariant metrics. Although they are all interesting in their own rights our main motivation for understanding bi-invariant word metrics was different.

1.C. A motivation and application. Let $(\mathrm{M}, \omega)$ be a symplectic manifold and let $\operatorname{Ham}(\mathrm{M}, \omega)$ denote the group of compactly supported Hamiltonian diffeomorphisms of $(\mathrm{M}, \omega)$. It admits a bi-invariant metric, called the Hofer metric (see Section 5.A for definition). This metric is known to have infinite diameter in many cases and no example of a symplectic manifold of positive dimension with the Hofer metric of finite diameter is known. We would like to understand the algebraic structure of $\operatorname{Ham}(M, \omega)$ in the sense of the following question.

Question 1.3. What are the finitely generated subgroups of the group of Hamiltonian diffeomorphisms of a symplectic manifold?

Corollary 1.4. Let $(\mathrm{M}, \omega)$ be a symplectic manifold. Let $\Gamma$ be a finite extension or a supergroup of finite index of a Chevalley group $\mathrm{G}_{\pi}\left(\Phi, \mathcal{O}_{\vee}\right)$. 
Then the image of a homomorphism

$$
\Gamma \rightarrow \operatorname{Ham}(\mathrm{M}, \omega)
$$

lies within a bounded distance from the identity with respect to the Hofer metric.

Proof. A homomorphism $\varphi: \Gamma \rightarrow \operatorname{Ham}(\mathrm{M}, \omega)$ is Lipschitz with respect to the bi-invariant word metric on $\Gamma$ and the Hofer metric on the group of Hamiltonian diffeomorphism (see Lemma 2.4). Since the bi-invariant word metric is bounded on $\Gamma$ according to Theorem 1.1 , the image of $\varphi$ is bounded in $\operatorname{Ham}(M, \omega)$.

1.D. Remarks. There are examples of nontrivial Hamiltonian actions of arithmetic lattices on closed symplectic manifolds (see Example 5.3. In all examples known to us, such an action factors through a compact group.

The well known result of Polterovich [20] states that there are no nontrivial Hamiltonian actions of certain lattices on symplectically hyperbolic manifolds [15].

Question 1.5. What are the bounded (and finitely generated) subgroups of $\operatorname{Ham}(\mathrm{M}, \omega)$ ?

Question 1.6. Is there a closed symplectic manifold $(\mathrm{M}, \omega)$ and a lattice $\Gamma$ in a semisimple Lie group such that there exists a homomorphism $\Gamma \rightarrow \operatorname{Ham}(\mathrm{M}, \omega)$ which does not factor through a compact group?

\section{PRELIMINARIES ON BI-INVARIANT WORD METRICS}

2.A. The word metric. Let $\Gamma$ be a group generated by a set $S \subset \Gamma$. The word norm on $\Gamma$ with respect to $S$ is defined by

$$
|g|_{S}=\min \left\{k \in \mathbf{N} \mid g=s_{i_{1}} \ldots s_{i_{k}} \text {, where } s_{i} \in \mathrm{S}\right\} \text {. }
$$

Suppose that $S=S^{-1}$. It is a standard fact that the above function satisfies the following properties for all elements $g, h \in \Gamma$.

(1) $|g|_{S} \geq 0$

(2) $|g|_{S}=0$ if and only if $g=1$

(3) $|g h|_{\mathrm{S}} \leq|g|_{\mathrm{S}}+|h|_{\mathrm{S}}$

(4) $\left|g^{-1}\right|_{S}=|g|_{S}$

Such a norm defines a right-invariant metric $d_{\mathrm{S}}$ on $\Gamma$ by

$$
d_{\mathrm{S}}(g, h)=\left|g h^{-1}\right|_{\mathrm{S}} \text {. }
$$


The metric is called the word metric associated with the generating set S. The geometry of such metrics for finitely generated groups has been a subject of extensive research during the last few decades originating in Gromov [10].

2.B. Bi-invariant word metrics. If the generating set $\mathrm{S}$ is invariant under the conjugation then so is the norm. That is, for all $g, h \in \Gamma$ we have

$$
\left|h g h^{-1}\right|_{\mathrm{s}}=|g|_{\mathrm{s}} \text {. }
$$

The induced metric is then bi-invariant. Let $S \subset \Gamma$ be a subset normally generating $\Gamma$. This means that $\Gamma$ is generated by

$$
\overline{\mathrm{S}}:=\bigcup_{g \in \Gamma} g S g^{-1} \text {. }
$$

The set $\bar{S}$ is invariant under the conjugation. If $S$ is a generating set then, since $\mathrm{S} \subset \overline{\mathrm{S}}$, we have

$$
|g|_{\bar{S}} \leq|g|_{S}
$$

for every $g \in \Gamma$. If $\Gamma$ is Abelian then the two norms coincide. In general, the bi-invariant norm is strictly smaller on some elements.

Remark 2.1. The basic properties presented in this section are elementary and can be found in the paper of Burago, Ivanov and Polterovich [2], where they investigate bi-invariant metrics on groups of diffeomorphisms of manifolds.

In general, not much is known for bi-invariant word metrics with an exception for the commutator length (see Section 3.B).

Example 2.2. Let $\Gamma=\mathrm{F}_{2}$ be a free group generated by two elements. Let $\mathrm{S}=\left\{x, x^{-1}, y, y^{-1}\right\}$. Observe that

$$
\left|y^{n} x y^{-n}\right|_{\bar{S}}=1 \text { and }\left|y^{n} x y^{-n}\right|_{S}=2 n+1 .
$$

Example 2.3. If $\Gamma$ has finitely many conjugacy classes then any biinvariant metric is bounded.

2.C. The Lipschitz property. A group $G$ is normally finitely generated if there exists a finite set $S \subset G$, such that $G$ is generated by all the conjugates of elements of $\mathrm{S}$.

Lemma 2.4. Let $\Gamma$ be a group normally generated by a finite set $\mathrm{S}=$ $\mathrm{S}^{-1}$. Let $\mathrm{G}$ be a group equipped with a bi-invariant norm \|\| . A homomorphism $\psi: \Gamma \rightarrow \mathrm{G}$ is Lipschitz. That is, there exists a positive constant $\mu \in \mathbf{R}$ such that

$$
\|\psi(g)\| \leq \mu|g|_{\bar{S}}
$$

for every $g \in \Gamma$. 
Proof. Let $\mu:=\max \{\|\psi(s)\| \mid s \in \mathrm{S}\}$ and let $g=s_{i_{1}}^{h_{1}} \ldots s_{i_{k}}^{h_{k}}$ be a word of minimal length showing that $|g|_{\bar{S}}=k$. Then

$$
\begin{aligned}
\|\psi(g)\| & =\left\|\psi\left(s_{i_{1}}\right)^{\psi\left(h_{1}\right)} \ldots \psi\left(s_{i_{k}}\right)^{\psi\left(h_{k}\right)}\right\| \\
& \leq \sum_{j=1}^{k}\left\|\psi\left(s_{i_{j}}\right)\right\| \\
& \leq \mu k=\mu|g|_{\bar{S}}
\end{aligned}
$$

Example 2.5. If $G$ is a simple group then it is normally generated by $\left\{g, g^{-1}\right\}$ for any $g \neq$ Id. Let us apply this to the group of Hamiltonian diffeomorphisms of a closed symplectic manifold. Let Id $\neq g \in$ $\operatorname{Ham}(\mathrm{M}, \omega)$ and let $\mathrm{S}=\left\{g, g^{-1}\right\}$. Then

$$
|f|_{\overline{\mathrm{S}}} \geq \frac{1}{\|g\|}\|f\|
$$

for every $f \in \operatorname{Ham}(\mathrm{M}, \omega)$ where $\|f\|$ denotes the Hofer norm.

The Lipschitz equivalence class of a bi-invariant word metric on a normally finitely generated group is well defined. And this class is maximal in the sense that any other bi-invariant metric is Lipschitz with respect to it. More precisely, the identity from the word metric to any other bi-invariant metric is Lipschitz. In particular, a normally finitely generated group $\mathrm{G}$ admits an unbounded bi-invariant metric if and only if the bi-invariant word metric is unbounded.

2.D. Convention. In what follows, we shall frequently abuse terminology by saying the bi-invariant word metric having in mind the Lipschitz equivalence class of such metrics. The notation $d_{\Gamma}$ will mean $d_{\overline{\mathrm{S}}}$ for some finite generating set $\mathrm{S} \subset \Gamma$.

Corollary 2.6. Let $\Gamma$ be a group normally generated by a finite set. If the associated bi-invariant word metric is bounded then every quotient of $\Gamma$ is bounded. In particular, the abelianisation $\Gamma /[\Gamma, \Gamma]$ is finite.

Example 2.7. Let $\Gamma=\mathbf{Z} / 2 \star \mathbf{Z} / 2$ be the infinite dihedral group. It is direct calculation that the bi-invariant word metric is bounded by 2 . On the other hand, $\Gamma$ contains an infinite cyclic subgroup of index two which has, of course, unbounded bi-invariant metric. This shows that the inclusion of a finite index (normal) subgroup is not Lipschitz. $\diamond$

Corollary 2.8. Let $\Gamma$ be a finitely generated group and let $\pi: \Gamma \rightarrow \Delta$ be a surjective homomorphism. If $s: \Delta \rightarrow \Gamma$ is a left inverse of $\pi$, that is, $s \circ \pi=\mathrm{Id}_{\Delta}$, then the bi-invariant word metric on $\Delta$ induced from $\Gamma$ is equivalent to the bi-invariant word metric $d_{\Delta}$. 
Corollary 2.9. If $\mathrm{F} \rightarrow \Gamma \rightarrow \Delta$ is a split extension with finite kernel then the quotient map is a bi-Lipschitz equivalence.

3. WHEN A BI-INVARIANT METRIC IS UNBOUNDED?

Most of the material presented in this section is known and standard, except possibly for the part about extensions.

\section{A. Distortion [10, Section 3].}

Let $\mathrm{G}$ be a group equipped with a norm \|\| . The translation length of an element $g \in \mathrm{G}$ is defined by

$$
\tau(g):=\lim _{n \rightarrow \infty} \frac{\left\|g^{n}\right\|}{n} .
$$

Lemma 3.1. For every $g \in \mathrm{G}$ we have $\mathrm{\tau}(g)=\inf _{n} \frac{\left\|g^{n}\right\|}{n}$.

Proof. First, observe that $0 \leq \frac{\left\|g^{n}\right\|}{n} \leq\|g\|$ and hence the above infimum exists. Let us denote this infimum by $\mu$. Let $\epsilon>0$ and let $m \in \mathbf{N}$ be such that

$$
\frac{\left\|g^{m}\right\|}{m}<\mu+\epsilon
$$

Choose an $n>\frac{m\|g\|}{\epsilon}$ and write it as $n=a m+b$, for some $a, b \in \mathbf{N}$ with $b<m$. Then we have

$$
\left\|g^{n}\right\|=\left\|g^{a m+b}\right\| \leq\left\|g^{a m}\right\|+\left\|g^{b}\right\| \leq a\left\|g^{m}\right\|+\left\|g^{b}\right\| .
$$

It follows that

$$
\frac{\left\|g^{n}\right\|}{n} \leq \frac{\left\|g^{m}\right\|}{m}+\epsilon<\mu+2 \epsilon .
$$

Since $\epsilon$ is arbitrary, this shows that

$$
\lim _{n \rightarrow \infty} \frac{\left\|g^{n}\right\|}{n} \leq \mu
$$

which finishes the proof.

An element $g \in \mathrm{G}$ is called distorted with respect to the norm \|\| if its translation length is equal to zero and undistorted otherwise.

Lemma 3.2. Let $\psi: \Gamma \rightarrow \mathrm{G}$ be a homomorphism. If $\psi(g)$ is undistorted then so is $g$. 
3.B. The commutator length [3]. Let $\mathrm{G}$ be a group. The commutator length

$$
\mathrm{cl}:[\mathrm{G}, \mathrm{G}] \rightarrow \mathbf{R}
$$

is defined to be the length of the shortest word expressing $g$ and consisting of commutators of elements from G. Notice that if $G=$ $[G, G]$ then the commutator length is a bi-invariant norm on $G$. Thus following observation is direct consequence of Lemma 2.4.

Lemma 3.3. Let $\Gamma$ be a perfect group generated by a finite set $\mathrm{S}=\mathrm{S}^{-1}$. Then there exists a constant $v>0$ such that

$$
\operatorname{cl}(g) \leq v|g|_{\bar{S}}
$$

for every $g \in \Gamma$. In other words, the bi-invariant word norm is Lipschitz with respect to the commutator length.

The stable commutator length of an element $g \in \mathrm{G}$ is defined as the translation length with respect to the commutator length. That is,

$$
\operatorname{scl}(g):=\lim _{n \rightarrow \infty} \frac{\operatorname{cl}\left(g^{n}\right)}{n} .
$$

The previous lemma has an immediate corollary.

Corollary 3.4. Let $\tau_{\bar{S}}$ denote the translation length of the $\overline{\mathrm{S}}$-word norm on a perfect group $\Gamma$ generated by a finite set $\mathrm{S}$. Then

$$
\operatorname{scl}(g) \leq v \tau_{\bar{S}}(g)
$$

for every $g \in \Gamma$ and the constant $v$ from Lemma 3.3 ,

3.C. Quasi-homomorphisms [17. Let G be a group. A quasi-homomorphism

$$
q: \mathrm{G} \rightarrow \mathbf{R}
$$

is a function such that there exist a constant $D \geq 0$ (called the defect of $q$ ) such that

$$
|q(g)-q(g h)+q(h)| \leq \mathrm{D}
$$

for every $g, h \in \mathrm{G}$. A quasi-homomorphism is called homogeneous if

$$
q\left(g^{n}\right)=n q(g)
$$

for every $g \in \mathrm{G}$ and every $n \in \mathbf{Z}$. If $q: \mathrm{G} \rightarrow \mathbf{R}$ is a quasi-homomorphism with defect $\mathrm{D}$ then the formula

$$
\widehat{q}(g):=\lim _{n \rightarrow \infty} \frac{q\left(g^{n}\right)}{n}
$$

defines a homogeneous quasi-homomorphism and we have

$$
|q(g)-\widehat{q}(g)| \leq \mathrm{D}
$$

for all $g \in \mathrm{G}$. Thus if $q$ is unbounded then so is its homogenisation and if $q$ is bounded then its homogenisation is identically zero. 
Lemma 3.5. [3, Theorem 2.70] Let $\mathrm{G}$ be a perfect group and let $q: \mathrm{G} \rightarrow \mathbf{R}$ be a homogeneous quasi-homomorphism. Then there exists a positive constant $\mathrm{C}>0$ such that

$$
|q(g)| \leq \mathrm{Cscl}(g)
$$

for every $g \in \mathrm{G}$.

Lemma 3.6. Let $q: \Gamma \rightarrow \mathbf{R}$ be a quasi-homomorphism of a group generated by a finite set $\mathrm{S}$. Then there exists a constant $\mathrm{C}>0$ such that

$$
|q(g)| \leq \mathrm{C}|g|_{\overline{\mathrm{S}}}
$$

for every $g \in \Gamma$.

Proof. Let $\mu:=\max \{|q(s)| \mid s \in \mathrm{S}\}$. Let $g \in \Gamma$ be of $\overline{\mathrm{S}}$-length equal to $k$. That is, $g=s_{i_{1}}^{h_{1}} \ldots s_{i_{k}}^{h_{k}}$. The following calculation follows directly from the quasi-homomorphism property of $q$.

$$
\begin{aligned}
|q(g)| & =\left|q\left(s_{i_{1}}^{h_{1}} \ldots s_{i_{k}}^{h_{k}}\right)\right| \\
& \leq(k-1) \mathrm{D}+\sum_{j=1}^{k}\left|q\left(s_{i_{j}}^{h_{j}}\right)\right| \\
& \leq(k-1) \mathrm{D}+\sum_{j=1}^{k}\left(2 \mathrm{D}+\left|q\left(s_{i_{j}}\right)\right|\right) \\
& \leq k \mathrm{D}+2 k \mathrm{D}+k \mu \\
& \leq(3 \mathrm{D}+\mu)|g|_{\bar{S}}
\end{aligned}
$$

Corollary 3.7. If $q: \Gamma \rightarrow \mathbf{R}$ is a homogeneous quasi-homomorphism on a group generated by a finite set $\mathrm{S}$ then there exists a constant $\mathrm{C}>0$ such that

$$
|q(g)| \leq \mathrm{C} \tau_{\overline{\mathrm{S}}}(g)
$$

for every $g \in \Gamma$. Consequently, if $q(g) \neq 0$ then $g$ is undistorted with respect to the bi-invariant word metric.

Example 3.8. If $\Gamma$ is a hyperbolic group then, due to Epstein and $\mathrm{Fu}-$ jiwara [8] the secound bounded cohomology group is infinite dimentional, therefore the comparison map $\mathrm{H}_{b}^{2}(\Gamma ; \mathbf{R}) \rightarrow \mathrm{H}^{2}(\Gamma ; \mathbf{R})$ has kernel, and as a corollary there exists nontrivial, thus unbounded quasimorphism on $\Gamma$ Consequently, the bi-invariant word metric on a nonelementary hyperbolic group is unbounded.

\section{D. Extensions.}

Proposition 3.9. Let $\mathrm{K} \stackrel{i}{\rightarrow} \widehat{\Gamma} \stackrel{\pi}{\rightarrow} \Gamma$ be an extension with bounded kernel K. Then $\widehat{\Gamma}$ is bounded if and only if the group $\Gamma$ is bounded. In particular, an extension of a bounded group by a finite group is bounded. 
Proof. If the extension is bounded then the quotient is bounded due to the Lipschitz property of the quotient homomorphism (see Section 2.C.

Suppose that the bi-invariant word metric of the quotient is bounded by $m$. Let $s: \Gamma \rightarrow \widehat{\Gamma}$ be a section such that $s(1)=1$. Let $\widehat{S}$ be a generating set of $\widehat{\Gamma}$ containing the image $s(\mathrm{~S})$ of the generating set of the quotient. Let $\kappa:=\max \left\{|i(k)|_{\hat{\Gamma}} \mid k \in \mathrm{K}\right\}$.

Let $\hat{g} \in \widehat{\Gamma}$ be any element. Let $\pi(\hat{g})=g_{1} \ldots g_{m}$. The following calculation yields the proof.

$$
\begin{aligned}
|\hat{g}|_{\widehat{\Gamma}} & =|s(\pi(\hat{g})) i(k)|_{\widehat{\Gamma}} \\
& =\left|s\left(g_{1} \ldots g_{m}\right) i(k)\right|_{\widehat{\Gamma}} \\
& =\left|s\left(g_{1}\right) \ldots s\left(g_{m}\right) i\left(k_{1}\right) \ldots i\left(k_{m}\right) i(k)\right|_{\widehat{\Gamma}} \\
& \leq m+\left|i\left(k_{1} \ldots k_{m} k\right)\right|_{\widehat{\Gamma}} \\
& \leq m+\kappa .
\end{aligned}
$$

Proposition 3.10. Let $\mathrm{Z} \stackrel{i}{\rightarrow} \widehat{\Gamma} \stackrel{\pi}{\rightarrow} \Gamma$ be a central extension associated with the class $0 \neq[c] \in \mathrm{H}^{2}(\Gamma, \mathbf{Z})$. If the cocycle $c$ is bounded then the image $i(\mathbf{Z}) \subset \widehat{\Gamma}$ is unbounded. Consequently, $\widehat{\Gamma}$ is unbounded.

Proof. Let B be a constant such that $|c(g, h)| \leq \mathrm{B}$ for every $g, h \in \Gamma$. Let $s: \Gamma \rightarrow \widehat{\Gamma}$ be a section such that $s(1)=1$. Let $S$ be a set normally generating $\Gamma$. Then its image $s(\mathrm{~S})$ normally generate $\widehat{\Gamma}$. We consider the word metrics with respect to these sets.

Suppose, on the contrary to the statement, that $i(\mathbf{Z}) \subset \widehat{\Gamma}$ is bounded. That is there exists a constant $\mathrm{C}$ such that $|i(k)|_{\Gamma} \leq \mathrm{C}$ for every $k \in \mathbf{Z}$. For any $k \in \mathbf{Z}$ we have the following equalities.

$$
\begin{aligned}
i(k) & =s\left(g_{1}\right) s\left(g_{2}\right) \ldots s\left(g_{m}\right) \\
& =s\left(g_{1} g_{2}\right) s\left(g_{3}\right) \ldots s\left(g_{m}\right) c\left(g_{1}, g_{2}\right) \\
& =s\left(g_{1} g_{2} \ldots g_{m}\right) c\left(g_{1}, g_{2}\right) c\left(g_{1} g_{2}, g_{3}\right) \ldots c\left(g_{1} g_{2} \ldots g_{m-1}, g_{m}\right) \\
& =c\left(g_{1}, g_{2}\right) c\left(g_{1} g_{2}, g_{3}\right) \ldots c\left(g_{1} g_{2}, g_{m-1}, g_{m}\right)
\end{aligned}
$$

It follows that $|k| \leq(m-1) \mathrm{B} \leq \mathrm{CB}$ which is a contradiction for $k \in \mathbf{Z}$ was chosen to be arbitrary.

Example 3.11. Let $\Gamma=\operatorname{Sp}(2 n, \mathbf{Z}) \subset \operatorname{Sp}(2 n ; \mathbf{R})$ be a lattice and let $\widehat{\Gamma}$ be the central extension that is the pullback of the universal cover $\widetilde{\mathrm{Sp}}(2 n ; \mathbf{R}) \rightarrow \mathrm{Sp}(2 n ; \mathbf{R})$ with respect to the inclusion of the lattice. 
It is known that this extension is associated with a bounded cohomology class and hence $\widehat{\mathrm{Sp}}(2 n ; \mathbf{Z})$ is unbounded, due to Proposition 3.10. On the other hand, the quotient $\operatorname{Sp}(2 n ; \mathbf{Z})$ is a Chevalley group and, according to Theorem 1.1, it is bounded.

\section{Bi-INVARIANT WORD METRICS ON CHEVALLEY GROUPS}

4.A. Chevalley groups. Let $\pi: \mathfrak{g} \rightarrow \mathfrak{g l}(\mathrm{V})$ be a representation of a complex semisimple Lie algebra and let $\mathcal{O}$ be a commutative ring with unit. Let $\Phi$ denotes the root system associated with a Cartan subalgebra $\mathfrak{h} \subset \mathfrak{g}$. With these data there are associated two groups

$$
\mathrm{E}_{\pi}(\Phi, \mathcal{O}) \subset \mathrm{G}_{\pi}(\Phi, \mathcal{O})
$$

called the elementary Chevalley group and the Chevalley group respectively. If $\mathcal{O}$ is a field then these groups coincide and are well understood [1, 5, 21]. The situation over rings is much more delicate [23]. Let us define the groups.

The elementary Chevalley group $\mathrm{E}_{\pi}(\Phi, \mathcal{O})$ is defined as the subgroup of the automorphism group $\operatorname{Aut}\left(\mathrm{V}_{\mathbf{Z}} \otimes_{\mathbf{Z}} \mathcal{O}\right)$ generated by elements of the form

$$
x_{\alpha}(t):=\exp \left(t \pi\left(x_{\alpha}\right)\right)
$$

where $\alpha \in \Phi$ is a root and $t \in \mathcal{O}$. Here, $\mathrm{V}_{\mathrm{Z}}$ is an admissible $\mathbf{Z}$-form of $\mathrm{V}$, that is, an integral lattice preserved by the representation, see Borel [1] for details.

Let $\mathrm{G} \subset \mathrm{G}(n, \mathbf{C})$ be a complex Lie group corresponding to the Lie algebra $\mathfrak{g}$, where the identification $\mathrm{GL}(\mathrm{V}) \cong \mathrm{GL}(n, \mathbf{C})$ is done via the basis of $\mathrm{V}_{\mathbf{Z}}$. This basis defines coordinate functions on $\mathrm{GL}(n, \mathbf{C})$ restrictions of which generate a Hopf subalgebra $Z[G] \subset[G]$ in the coordinate ring for $\mathrm{G}$. The Chevalley group is defined to be an affine group scheme over the integers

$$
\mathrm{G}_{\pi}(\Phi, \mathcal{O}):=\operatorname{Hom}(\mathbf{Z}[\mathrm{G}], \mathcal{O}) .
$$

Remark 4.1. Both definitions above depend on the choice of the admissible $\mathbf{Z}$-form $\mathrm{V}_{\mathbf{Z}}$. This choice is not mentioned in our abused notation.

It is not difficult to see that there is an inclusion $\mathrm{E}_{\pi}(\Phi, \mathcal{O}) \subset \mathrm{G}_{\pi}(\Phi, \mathcal{O})$. Moreover, if $\mathrm{G}$ is of rank at least two and $\mathcal{O}=\mathcal{O}_{\mathrm{V}}$ is the ring of $\mathrm{V}$ integers in a number field then the two groups coincide (see Tavgen' [22, Lemma 4]).

Lemma 4.2. [5, Theorem 5.2.2] The generators $x_{\alpha}(t) \in \mathrm{E}_{\pi}(\Phi, \mathcal{O})$ satisfy the following commutation relations,

$$
\left[x_{\alpha}(k), x_{\beta}(l)\right]=\prod_{i, j} x_{i \alpha+j \beta}\left(\mathrm{C}(-l)^{i} k^{j}\right)
$$


where $i, j$ are positive integers such that $i \alpha+j \beta$ is a root and $\mathrm{C}$ is an integer such that $|\mathrm{C}| \leq 3$. The product is taken in the order of increasing $i+j$.

4.B. Proof of Theorem 1.1. We shall prove the statement for the elementary Chevalley group. Since it is of finite index in the Chevalley group the result follows for the latter and for a general $\Gamma$ as in the statement of theorem as well.

We shall show that there exists a positive number $m \in \mathbf{R}$ such that for every element $g \in \mathrm{E}_{\pi}\left(\Phi, \mathcal{O}_{\bigvee}\right)$ and every $n \in \mathbf{Z}$ we have $\left|g^{n}\right| \leq m$. The first step is to prove this claim for an element of the form $x_{\alpha}(r) \in$ $\mathrm{E}_{\pi}\left(\Phi, \mathcal{O}_{\vee}\right)$, where $\alpha \in \Phi$ is a root and $r \in \mathcal{O}_{\vee}$.

There exist a subsystem $\Psi \subset \Phi$ of rank two isomorphic to either $\mathbf{A}_{2}$ or $\mathbf{B}_{2}$ and containing $\alpha$. Indeed, there is a subsystem of rank two containing $\alpha$ and it follows from the simplicity and the higher rank that this system has to be simple of rank two, that is one of $\mathbf{A}_{2}, \mathbf{B}_{2}, \mathbf{G}_{2}$. It is then easy to see that each root in $\mathbf{G}_{2}$ is contained in some $\mathbf{A}_{2}$.

Observe that there exists $\beta, \gamma \in \Psi$ such that $\alpha=\beta+\gamma$ and no other positive combination of $\beta$ and $\gamma$ is a root. It follows from Lemma 4.2 that

$$
x_{\alpha}(-\mathrm{C} k r)=\left[x_{\beta}(k r), x_{\gamma}(1)\right]
$$

where $k \in \mathbf{Z}$ and $\mathrm{C}= \pm 1, \pm 2$ or \pm 3 . Thus we obtain that

$$
\left|x_{\alpha}(\mathrm{Cr})^{k}\right|=\left|x_{\alpha}(r)^{\mathrm{C} k}\right| \leq 2
$$

which implies that the cyclic subgroup generated by $x_{\alpha}(r)$ stays within a bounded distance from the identity.

It is a result of Tavgen' [22] that the group $\mathrm{E}_{\pi}\left(\Phi, \mathcal{O}_{\mathrm{V}}\right)$ has bounded generation with respect to the set of elements of the form $x_{\alpha}(r)$. That is, there is a constant $\mathrm{B} \in \mathbf{N}$ such that every $g \in \mathrm{E}_{\pi}\left(\Phi, \mathcal{O}_{\vee}\right)$ is a product of at most B elements of the form $x_{\alpha}(r)$.

Let $\rho_{1}, \ldots, \rho_{n} \in \mathcal{O}_{\mathrm{V}}$ be elements such that there is an isomorphism $\mathcal{O}_{V} \cong \mathbf{Z} \rho_{1} \oplus \ldots \oplus \mathbf{Z} \rho_{n}$ of abelian groups. Let $\mathrm{S}:=\left\{x_{\alpha}\left(\rho_{i}\right)\right\}$ be a set of generators of $\mathrm{G}_{\pi}\left(\Phi, \mathcal{O}_{\vee}\right)$ and let $\mu$ be a number such that

$$
\left|x_{\alpha}\left(\rho_{i}\right)^{k}\right|_{\bar{S}} \leq \mu
$$

for all $1 \leq i \leq n, \alpha \in \Phi$ and $k \in \mathbf{Z}$. 
Putting the two results together we obtain the following estimate for every $g \in \mathrm{E}_{\pi}\left(\Phi, \mathcal{O}_{\vee}\right)$.

$$
\begin{aligned}
|g|_{\overline{\mathrm{S}}} & =\left|x_{\alpha_{1}}\left(r_{1}\right)^{k_{1}} \ldots x_{\alpha_{l}}\left(r_{l}\right)^{k_{l}}\right|_{\overline{\mathrm{S}}} \\
& =\left|x_{\alpha_{1}}\left(\rho_{1}\right)^{k_{11}} \ldots x_{\alpha_{1}}\left(\rho_{n}\right)^{k_{1 n}} \ldots x_{\alpha_{l}}\left(\rho_{1}\right)^{k_{l 1}} \ldots x_{\alpha_{l}}\left(\rho_{n}\right)^{k_{l n}}\right|_{\overline{\mathrm{S}}} \\
& =\left|x_{\alpha_{1}}\left(\rho_{1}\right)^{k_{11}}\right|_{\overline{\mathrm{S}}}+\ldots+\left|x_{\alpha_{l}}\left(\rho_{n}\right)^{k_{l n}}\right|_{\overline{\mathrm{S}}} \\
& \leq \mu l n \leq \mu \mathrm{B} n .
\end{aligned}
$$

Corollary 4.3. Let $\Gamma$ be a group as in Theorem 1.1, Then

(1) if $\Gamma$ is perfect then the commutator length is bounded on $\Gamma$ and hence its stable commutator length is zero;

(2) if $\Gamma$ is generated by torsion elements then the torsion length is bounded;

(3) every quasi-homomorphism $q: \Gamma \rightarrow \mathbf{R}$ is bounded.

4.C. Properties and examples of Chevalley groups. Suppose that $\mathrm{G}$ is an algebraic group defined over $\mathcal{O}$. It is clear from the definition of (elementary) Chevalley group that we have inclusions

$$
\mathrm{E}_{\pi}\left(\Phi, \mathcal{O} \vee \subset \mathrm{G}_{\pi}(\Phi, \mathcal{O} \mathrm{v}) \subset \mathrm{G}(\mathcal{O})\right.
$$

In what follows we list various examples of bounded groups. Notice that each example provides more groups by taking finite extensions, quotients and finite index supergroups.

Example 4.4. The special linear group $\operatorname{SL}(n, \mathbf{Z})$ for $n \geq 3$ is bounded because it is the Chevalley group $\mathrm{G}\left(\mathbf{A}_{n-1}, \mathbf{Z}\right)$. It is a non-uniform lattice in $\mathrm{SL}(n, \mathbf{Z})$.

Example 4.5. The special linear group $\operatorname{SL}(n, Z[\sqrt{2}])$ for $n \geq 3$ is bounded because it is the Chevalley group $\mathrm{G}\left(\mathbf{A}_{n-1}, \mathbf{Z}[\sqrt{2}]\right)$. It is a non-uniform lattice in $\operatorname{SL}(n, \mathbf{Z}) \times \operatorname{SL}(n, \mathbf{Z})$.

Example 4.6. The special linear group $\operatorname{SL}(n, \mathbf{Z}[i])$ for $n \geq 3$ is bounded because it is the Chevalley group $\mathrm{G}\left(\mathbf{A}_{n-1}, \mathbf{Z}[i]\right)$. It is a non-uniform lattice in $\operatorname{SL}(n, \mathbf{C})$.

Example 4.7. Let B be a quadratic form represented by the matrix with ones on the antidiagonal and zeros elsewhere. The associated orthogonal group $\mathrm{SO}(n, n+1, \mathbf{Z})$ for $n \geq 2$ is bounded because it is the Chevalley group $\mathrm{G}\left(\mathbf{B}_{n}, \mathbf{Z}\right)$. It is a non-uniform lattice in $\mathrm{SO}(n, n+1)$. A similar example exists for the root system $\mathbf{D}_{n}$.

Example 4.8. For the root system $\mathbf{C}_{n}$ we obtain that the Chevalley group $\mathrm{G}\left(\mathbf{C}_{n}, \mathbf{Z}\right)$ is equal to $\operatorname{Sp}(2 n ; \mathbf{Z})$ and it is a non-uniform lattice in the split real form $\operatorname{Sp}(2 n ; \mathbf{R})$. 


\section{HAMILTONIAN REPRESENTATIONS}

5.A. The Hofer metric [11, 19]. Let $(\mathrm{M}, \omega)$ be a symplectic manifold. That is, $M$ is a smooth manifold and $\omega$ is a closed and non-degenerate two-form.

Let $\mathrm{H}: \mathrm{M} \times \mathbf{R} \rightarrow \mathbf{R}$ be a smooth function. It follows from he nondegeneracy of the symplectic form that the time-dependent vector field satisfying the identity

$$
\mathrm{t}_{t} \omega=d \mathrm{H}(, t)
$$

is well defined. Moreover, according to the closeness of the symplectic form the flow $f_{t}$ of this vector field preserves the symplectic form, $f_{t}^{*} \omega=\omega$.

A diffeomorphism obtained this way is called Hamiltonian and the group of Hamiltonian diffeomorphisms is denoted by $\operatorname{Ham}(M, \omega)$.

Let $f \in \operatorname{Ham}(\mathrm{M}, \omega)$ be a compactly supported Hamiltonian diffeomorphism generated by a function $\mathrm{H}$. The following formula

$$
\|f\|:=\inf _{\mathrm{H}} \int_{0}^{1} \operatorname{osc} \mathrm{H}(, t) d t
$$

defines a bi-invariant norm called the Hofer norm. The induced biinvariant metric is also called the Hofer metric. It is known to be unbounded in many cases [7, 18].

\section{B. Ad hoc examples of Hamiltonian representations.}

Example 5.1. Let $\Delta$ be a graph and let $\Gamma_{\Delta}$ be the right-angled Artin group associated with $\Delta$. That is, $\Gamma_{\Delta}$ is generated by the vertices $v_{1}, \ldots, v_{m}$ of $\Delta$ modulo the following commutation relations $\left[v_{i}, v_{j}\right]=1$ if and only if $\left\{v_{i}, v_{j}\right\}$ is not an edge of $\Delta$.

Let $\left\{\mathrm{U}_{i}\right\} \subset \mathrm{M}$ be a family of open subset such that their incidence graph is isomorphic to $\Delta$. Let $f_{i} \in \operatorname{Ham}(\mathrm{M}, \omega)$ be a diffeomorphism supported in $\mathrm{U}_{i}$. The map $\Gamma_{\Delta} \rightarrow \operatorname{Ham}(\mathrm{M}, \omega)$ defined by $v_{i} \mapsto f_{i}$ is clearly a homomorphism.

Such representations provide sometimes less obvious representations due to the fact that right-angled Artin groups contain many interesting subgroups [12]. The injectivity of certain Hamiltonian actions of right-angled Artin groups on two-dimensional sphere has been proven by M.Kapovich [13].

Example 5.2. Suppose that $\operatorname{Ham}(M, \omega)$ contains two tori $T_{1}$ and $T_{2}$ with nonempty intersection $\mathrm{T}_{1} \cap \mathrm{T}_{2} \neq \varnothing$. By choosing a finite number of generators $f_{1}, \ldots, f_{n} \in \mathrm{T}_{1} \cup \mathrm{T}_{2}$ we obtain a representation

$$
\mathrm{A} \star_{\mathrm{B}} \mathrm{C} \rightarrow \operatorname{Ham}(\mathrm{M}, \omega)
$$


of a finitely presented nontrivial amalgamated product of two Abelian groups. For example, if $(M, \omega)$ is a Hirzebruch surface then, in general, $\operatorname{Ham}(M, \omega)$ contains two dimensional tori satisfying the above assumption [14]. This way we obtain examples of Hamiltonian actions of finitely presented groups which do not extend to an action of a compact group.

Notice that in the above examples the groups are unbounded with respect to the bi-invariant word metric (with few obvious exceptions). Recall that Corollary 1.4 states that the image of a Hamiltonian action $\varphi: \Gamma \rightarrow \operatorname{Ham}(\mathrm{M}, \omega)$, where $\Gamma$ is either a finite extension or a supergroup of finite index of a Chevalley group is bounded. The only examples of bounded subgroups of the group of Hamiltonian diffeomorphisms of a closed symplectic manifold we know are subgroups of compact Lie groups.

Example 5.3. Let $\mathrm{H} \subset \operatorname{Ham}(\mathrm{M}, \omega)$ be the inclusion of a connected Lie group. According to a theorem of Delzant [6], if $\mathrm{H}$ is semisimple then it is compact. Let $\mathrm{G}$ be a non-compact connected semisimple Lie group and let $\Gamma \subset \mathrm{G} \times \mathrm{H}$ be an irreducible lattice. For example, let $\mathrm{J}_{p . q}$ be the diagonal matrix with the first $p$ entries equal to one and the last $q$ entries equal to $-\sqrt{2}$, where $p \geq q>1$. Let

$$
\Gamma:=\left\{\mathrm{A} \in \mathrm{SL}(p+q, \mathbf{Z}[\sqrt{2}]) \mid \mathrm{AJA}^{\mathrm{T}}=\mathrm{J}\right\}
$$

It is known that $\Gamma$ is a cocompact irreducible lattice in $\mathrm{SO}(p, q) \times$ $\mathrm{SO}(p+q)$. Taking the composition of the inclusion and the projection onto the second factor we obtain and a highly nontrivial Hamiltonian action of $\Gamma$ on coadjoint orbits of $\mathrm{SO}(2 n)$. Notice, however that $\Gamma$ is not a Chevalley group.

In the noncompact case the situation is different.

Example 5.4. Let $\mathbf{D}(r) \subset \mathbf{R}^{2 n}$ be an open $2 n$-dimensional disc of radius $r$ centred at the origin. It induces an inclusion

$$
\operatorname{Ham}\left(\mathbf{D}(r), \omega_{0}\right) \subset \operatorname{Ham}\left(\mathbf{R}^{2 n}, \omega_{0}\right) .
$$

The Hofer diameter of $\operatorname{Ham}\left(\mathbf{D}(r), \omega_{0}\right)$ is infinite since it can be estimated from below by the absolute value of the Calabi homomorphism.

On the other hand, the above inclusion is highly distorted. It is a result of Sikorav (Theorem 5 in Chapter 5 of Hofer-Zehnder [11]) that $\operatorname{Ham}\left(\mathbf{D}(r), \omega_{0}\right)$ is within a bounded distance from the identity with respect to the Hofer metric on $\operatorname{Ham}\left(\mathbf{R}^{2 n}\right)$. 


\section{C. Other restrictions on actions of lattices.}

Example 5.5. Let $\Gamma$ be a irreducible non-uniform lattice in a semisimple Lie group of higher rank. It is a result of Polterovich [20] that if $(\mathrm{M}, \omega)$ is a closed symplectically hyperbolic manifold then there is no non-trivial homomorphism $\Gamma \rightarrow \operatorname{Ham}(\mathrm{M}, \omega)$. It would be interesting to know if there are nontrivial bounded subgroups in $\operatorname{Ham}(\mathrm{M}, \omega)$ where $(\mathrm{M}, \omega)$ is symplectically hyperbolic.

Our final comment is concerned with actions supported on a proper subset of a manifold. In such cases there are severe restrictions coming from Thurston Stability as presented by Franks in [9]. It implies that there is no chance for embedding a lattice $\Gamma \subset \mathrm{G}$ in a semisimple Lie group of higher rank into a compactly supported diffeomorphisms of a non-compact manifold.

Proposition 5.6. Let $\Gamma$ be a finitely generated group. Let $\Gamma \rightarrow \operatorname{Diff}(\mathrm{M})$ be a smooth effective action with support strictly smaller than $\mathrm{M}$. Then either $\Gamma$ is trivial or it admits a nontrivial homomorphism $\Gamma \rightarrow \mathbf{R}$.

Acknowledgements. The authors would like to thank Dave Benson, Meinolf Geck, Tadeusz Januszkiewicz, Colin Maclachlan, Nicolas Monod, Leonid Polterovich, Geoff Robinson and Alain Valette for helpful discussions. The authors thank Kamil Duszenko, Denis Osin, and Yehuda Shalom for pointing out mistakes in an earlier version of the paper.

Ś.Gal is partially supported by Polish MNiSW grant N N201 541738, Swiss NSF Sinergia Grant CRSI22-130435, and by the European Research Council (ERC) grant of Goulnara Arzhantseva (grant agreement 259527)

\section{REFERENCES}

[1] Seminar on Algebraic Groups and Related Finite Groups. (Held at The Institute for Advanced Study, Princeton, N. J., 1968/69). Lecture Notes in Mathematics, Vol. 131. Springer-Verlag, Berlin, 1970.

[2] Burago, D., Ivanov, S., And Polterovich, L. Conjugation-invariant norms on groups of geometric origin. In Groups of diffeomorphisms, vol. 52 of Adv. Stud. Pure Math. Math. Soc. Japan, Tokyo, 2008, pp. 221-250.

[3] Calegari, D. scl, vol. 20 of MSJ Memoirs. Mathematical Society of Japan, Tokyo, 2009.

[4] Calegari, D., AND Zhuang, D. Stable w-length. arXiv:1008.2219.

[5] CARTER, R. W. Simple groups of Lie type. John Wiley \& Sons, London-New York-Sydney, 1972. Pure and Applied Mathematics, Vol. 28.

[6] Delzant, T. Sous-algebres de dimension finie de l'algebre des champs hmiltoniens.

[7] Entov, M., And Polterovich, L. Calabi quasimorphism and quantum homology. Int. Math. Res. Not., 30 (2003), 1635-1676. 
[8] Epstein, D. B. A., And Fujiwara, K. The second bounded cohomology of wordhyperbolic groups. Topology 36, 6 (1997), 1275-1289.

[9] FRANKs, J. Distortion in groups of circle and surface diffeomorphisms. In Dynamique des difféomorphismes conservatifs des surfaces: un point de vue topologique, vol. 21 of Panor. Synthèses. Soc. Math. France, Paris, 2006, pp. 35-52.

[10] Gromov, M. Asymptotic invariants of infinite groups. In Geometric group theory, Vol. 2 (Sussex, 1991), vol. 182 of London Math. Soc. Lecture Note Ser. Cambridge Univ. Press, Cambridge, 1993, pp. 1-295.

[11] Hofer, H., AND ZeHNDER, E. Symplectic invariants and Hamiltonian dynamics. Birkhäuser Advanced Texts: Basler Lehrbücher. [Birkhäuser Advanced Texts: Basel Textbooks]. Birkhäuser Verlag, Basel, 1994.

[12] Januszkiewicz, T., AND ŚwişTKowski, J. Commensurability of graph products. Algebr. Geom. Topol. 1 (2001), 587-603 (electronic).

[13] KAPOvich, M. RAAGs in Ham. arXiv.org:1104.0348.

[14] KARSHON, Y. Maximal tori in the symplectomorphism groups of Hirzebruch surfaces. Math. Res. Lett. 10, 1 (2003), 125-132.

[15] KȨDRA, J. Symplectically hyperbolic manifolds. Differential Geom. Appl. 27, 4 (2009), 455-463.

[16] Kотsсніск, D. Quasi-homomorphisms and stable lengths in mapping class groups. Proc. Amer. Math. Soc. 132, 11 (2004), 3167-3175.

[17] Kотsснick, D. What is... a quasi-morphism? Notices Amer. Math. Soc. 51, 2 (2004), 208-209.

[18] Ostrover, Y. A comparison of Hofer's metrics on Hamiltonian diffeomorphisms and Lagrangian submanifolds. Commun. Contemp. Math. 5, 5 (2003), 803-811.

[19] Polterovich, L. The geometry of the group of symplectic diffeomorphisms. Lectures in Mathematics ETH Zürich. Birkhäuser Verlag, Basel, 2001.

[20] Polterovich, L. Growth of maps, distortion in groups and symplectic geometry. Invent. Math. 150, 3 (2002), 655-686.

[21] SteinberG, R. Lectures on Chevalley groups. Yale University, New Haven, Conn., 1968. Notes prepared by John Faulkner and Robert Wilson.

[22] TAvGEN', O. I. Bounded generability of Chevalley groups over rings of S-integer algebraic numbers. Izv. Akad. Nauk SSSR Ser. Mat. 54, 1 (1990), 97-122, 221222.

[23] Vavilov, N., And Plotkin, E. Chevalley groups over commutative rings. I. Elementary calculations. Acta Appl. Math. 45, 1 (1996), 73-113.

ŚRG - UniWERSYTEt WrocŁaWsKI \& UNIVERSitÄT WiEN

E-mail address: sgal@math.uni.wroc.pl

JK - University of AberdeEn \& Uniwersytet SZCZECIŚNSKI

E-mail address: kedra@abdn.ac.uk 